\title{
Chapter 9 \\ From Arts to Action: Project SHINE as a Case Study of Engaging Youth in Efforts to Develop Sustainable Water, Sanitation, and Hygiene Strategies in Rural Tanzania and India
}

\author{
Anise Gold-Watts, Marte Hovdenak, Aruna Ganesan, and Sheri Bastien
}

\subsection{Arts-Based Engagement in Research: Project SHINE}

Through creative and collaborative processes, arts-based methods have been used to varying degrees within health promotion research and evidence-based public health interventions (Delgado 2015; Israel et al. 2005; Leavy 2015; Chap. 1, this volume). Art is fundamentally communicative and expressive (Barone and Eisner 2012), thus capable of inciting collaboration, perspective, emotion, dialogue, and/or action. Studies have demonstrated that arts-based methods, when applied to research approaches, can be used to develop community partnerships, to manage sensitive issues, to involve community members in the interpretation of data, and as a dissemination tool to communicate knowledge and empower participants to catalyze change in their communities (Delgado 2015; Lambert and Hessler 2018; Lohan et al. 2015; McEwan et al. 2013; Vindrola-Padros et al. 2016). Additionally, artsbased methods hold the potential to promote communication beyond language and

\footnotetext{
A. Gold-Watts $(\square)$

Department of Public Health Science, Faculty of Landscape and Society, Norwegian University of Life Sciences, Ås, Norway

e-mail: anise.gold-watts@nmbu.no

M. Hovdenak

Department of Health Promotion and Development, University of Bergen, Bergen, Norway

A. Ganesan

Sri Narayani Vidyalaya School, Vellore, India

\author{
S. Bastien \\ Department of Public Health Science, Faculty of Landscape and Society, Norwegian \\ University of Life Sciences, Ås, Norway \\ Department of Community Health Sciences, Cumming School of Medicine, University of
} Calgary, Calgary, AB, Canada
} 
encourage close collaboration between academic researchers and community participants throughout the research process.

There are several different art forms often used in arts-based research, including the performing arts such as music, spoken word, and drama; visual arts such as painting, drawing, design, and crafts; community and cultural affairs such as festivals, fairs, and events; literature, poetry, and creative writing; and online, digital, or electronic arts (Cahnmann-Taylor and Siegesmund 2018; Delgado 2015; Lambert and Hessler 2018). These arts-based methods can be applied to research approaches in various ways (Coemans and Hannes 2017; Wang et al. 2017), thus reflections on the application and effects of arts-based methods are key in continuing to develop this field of research. The arts-based methods discussed in this chapter were used as tools of inquiry, awareness raising, adaptation, and knowledge translation. Knowledge translation is a process of contextualization and application (Campbell 2012; Graham et al. 2006), which according to the Canadian Institutes of Health Research promotes the "synthesis, dissemination, exchange, and ethically-sound application of knowledge" to improve health outcomes (Straus et al. 2009). Throughout this chapter, we will reflect on several distinct features of arts-based methods as (1) a tool for awareness raising, knowledge translation, and action and (2) a process of promoting meaningful and equitable partnerships in communitybased participatory research (CBPR) within the frame of Project SHINE (Sanitation and Hygiene INnovation in Education) in India and Tanzania.

Implemented in two countries, Project SHINE is a school-based intervention that aims to improve water, sanitation, and hygiene (WASH)-related knowledge, attitudes, and behaviors among students, teachers, and local communities. In addition to supporting participatory approaches in the classroom, SHINE encourages youth to become health promoters and change-makers within their communities through the development of life and leadership skills. Unlike other WASH interventions that may employ techniques that promote "shame" or "social stigma" as a means for behavior change (Bartram et al. 2012; Kar and Chambers 2008; Pattanayak et al. 2009), Project SHINE uses an assets-based approach that incorporates CBPR and various arts-based methods throughout all phases of the research as a tool for awareness raising, knowledge translation, and action to promote meaningful engagement for participants and community members to result in synergy (for further background on the concept of synergy, see Chap. 21).

Throughout the intervention in Tanzania, art was used as a means to facilitate dialogue and promote knowledge-sharing and meaningful participant engagement. Arts-based health promotion activities such as open defecation mapping, a sanitation mural, a sanitation time capsule, digital stories, songs, and a sanitation science fair were incorporated throughout the intervention. These activities were used in formative research, implementation, and knowledge translation phases of the intervention and promoted participant inquiry and reflection, youth mobilization, and leadership focused on water, sanitation, and hygiene in the community.

During our adaptation to the southern Indian cultural context, the medium of photography was incorporated into the formative research phase through the CBPR process of photovoice. Here, we sought to develop a supportive research 
environment in which participants were positioned as experts and photography was used to stimulate knowledge production, participation, and co-learning (Wang and Burris 1997). This arts-based method enabled student participants to identify and represent (Wang et al. 1998) their communities and lived experiences in order to help us understand WASH-related contextual factors and facilitate the interpretation, adaption, and translation of Project SHINE to the local cultural context.

\subsection{SHINE Tanzania: An Application of the Arts to Create a Broad Platform for Youth Expression and Engagement}

SHINE Tanzania was developed and implemented in 2014 (see Fig. 9.1) as a pilot study to engage students in two secondary schools and the wider community in the development and evaluation of strategies to improve water, sanitation, and hygiene. The focus of the intervention was grounded in community concerns regarding the impact of parasitic infection on child health and from local hospital records, which indicated that fecal-oral transmitted diseases including helminth infections and protozoa are prevalent in the region. SHINE Tanzania was first implemented in a Maasai pastoralist community in the Ngorongoro Conservation Area (NCA) in rural and remote Tanzania. It was a collaboration between academic researchers and students from the University of Calgary, Canada, and the Catholic University of Health and Allied Sciences, Tanzania, as well as through a long-term partnership with communities of Maasai pastoralists in the NCA. The transdisciplinary research team included members with expertise in diverse fields-ranging from education, psychology, and anthropology to global health, veterinary medicine, and bioengineering — who work within a One Health paradigm (Zinsstag et al. 2011), which focuses on the interrelationships between humans, animals, and the environment. The study design has been described in full elsewhere (Bastien et al. 2016), as has the process and outcome evaluation of the intervention (Hetherington et al. 2017). In the intervention, a sequenced suite of participatory arts-based methods were used, including: open defecation mapping (9.2.1), a sanitation mural (9.2.3.1), a time capsule (9.2.3.2), songs, the capstone event of the sanitation science fair (9.2.2), and subsequent digital stories (9.2.3.3) to engage youth in reflecting on their experience with SHINE.

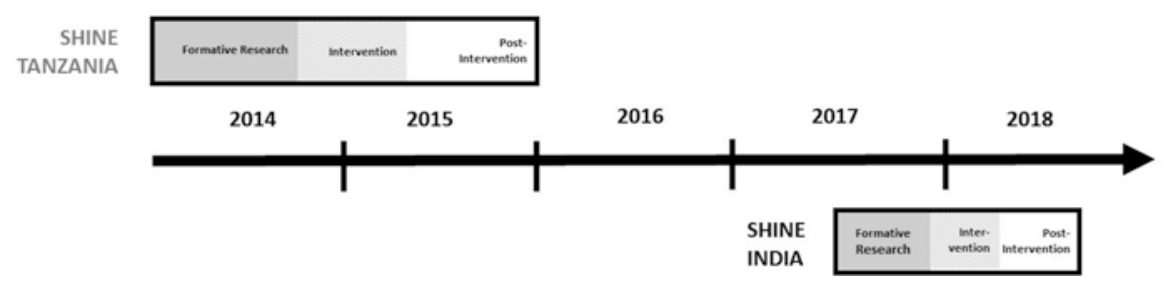

Fig. 9.1 Project SHINE timelines 


\subsubsection{Open Defecation Mapping}

During the formative research phase, which involved qualitative research both in schools and in the wider community (see for instance Henderson et al. 2016), we wanted to engage youth in an icebreaker activity that would raise the potentially sensitive issue of open defecation in a non-threatening way. We refrained from asking about individual practices and instead asked about how the community and physical environment were structured and what common WASH practices were. This approach simultaneously contributed to our understanding of the broader community context and of WASH behaviors. Using poster paper and markers, we invited the students to draw a map of their village, indicating bomas (houses) where people and animals sleep, water sources, and places where animals and people commonly defecate. We then asked them a series of questions, such as what different water bodies are used for (washing clothes, drinking water for humans and livestock), what happens when it rains, if flies are common in the area, how they understand contamination, and what they perceive to be "safe" water. This led to a reflective discussion on health and how participants understand the causes of diarrheal disease. This exercise also provoked laughter among the students, who were at first tentative to draw on the paper but once they understood the purpose became animated and engaged.

\subsubsection{Sanitation Science Fair-Culturally Relevant Knowledge Translation Strategies}

The development and evaluation of the sanitation science fair has been described in detail elsewhere (Bastien et al. 2017); however, in brief, it was designed to engage and empower youth and communities in the development and evaluation of locally relevant and sustainable health promotion strategies to improve sanitation and hygiene. Student teams were separated into three broad categories (e.g., water, sanitation, and hygiene) and with the guidance of biology teachers and the research team developed sanitation science fair projects, which were showcased at a One Health sanitation science fair in November 2014. Approximately 400 Form 3 students participated from two secondary schools. Then, an evaluation team consisting of a broad cross-section of community members was tasked with identifying promising projects that were particularly relevant given the community context and which held potential for social entrepreneurship. The wider community was invited to attend the science fair, and there were between 500 and 1000 in attendance at the two schools, which included representatives of the Pastoralist Council (local government), local education authorities, traditional leaders, and out-of-school youth.

Each project included a knowledge translation component, which focused on the public health implications of the science fair project findings for the wider 
community. Through discussions with our local community partners regarding what would be culturally relevant techniques to transmit knowledge in the local Maasai context, students used approaches such as songs, which were an engaging means to involve students actively and reflexively in applying and translating what they had learned throughout the SHINE intervention to the wider community. One such example is a team of students that developed an experiment to test how many folds in a cloth were needed in order to filter water. Based on their findings, they developed a song to teach to women that could be sung while collecting water.

\subsubsection{SHINE Arts-Based School and Community Event}

As part of the knowledge translation activities post-intervention, a one-day event was organized at the school to engage youth and community members in a series of arts-based approaches to elicit perspectives on what participating in Project SHINE meant to them. The day was organized around a series of facilitated stations whereby youth, teachers, and community stakeholders-including the evaluation team from the sanitation science fair-circulated to each station to participate in an activity and contribute to broadening our understanding of the impact of the study from their perspective. The stations included: (1) posters with visuals developed by the Bachelor of Health Sciences students from the University of Calgary to depict the main findings of the study and to gather perspectives to help interpret the findings; (2) a sanitation mural whereby participants were invited to paint what their experience with SHINE meant to them; (3) a Foldscope station that provided another opportunity to prepare and look at locally collected samples of water, soil, and plants collected from the schoolyard; and (4) a digital storytelling station. Highlights from the selected facilitated stations are elaborated on below.

\subsubsection{Sanitation Mural}

The sanitation mural was used as a visual method through the project to elicit student perspectives on what participating in Project SHINE meant to them. We provided a white sheet and a selection of paints and brushes on a table and asked participants to illustrate by any means what their SHINE experience meant to them (Figs. 9.2 and 9.3). The canvas was ceremoniously initiated by a traditional leader and member of the SHINE evaluation team giving a speech and painting the first stroke. Students and the wider community used words, phrases, and pictures to highlight the essence of the project, and it was later showcased in the school and formed part of the time capsule. In discussion with students and teachers, it became clear that painting was not an activity they had engaged in previously, but one which they enjoyed tremendously. 


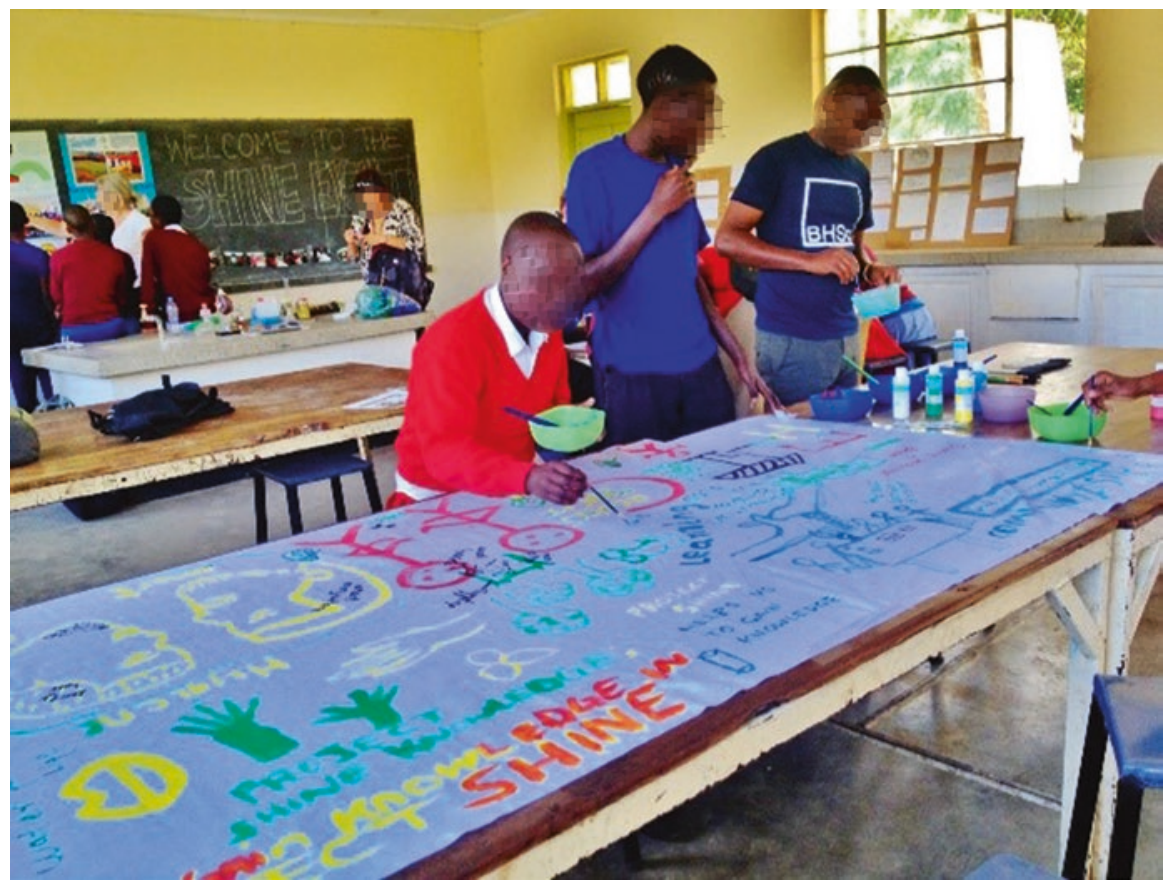

Fig. 9.2 Students and teachers collaborating on the development of a sanitation mural

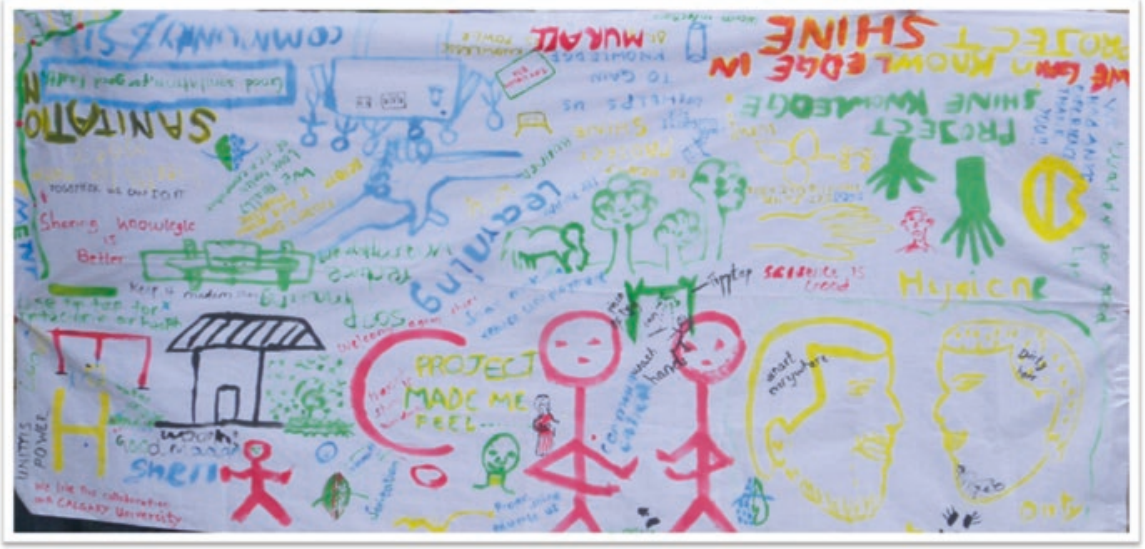

Fig. 9.3 Sanitation mural from SHINE Tanzania 


\subsubsection{Time Capsule}

A time capsule is a historic cache of information or items that can be used both as a memory that participants can look back and reflect on at a later date and as a method of communicating with people in the future, to share perspectives, hopes, and dreams from an earlier time period. Within Project SHINE, this assignment was given in a civics class and framed as a means to get students to think about what they envision in the future for their community and the changes they might like to see in the next 5 years. The assignments consisted of a worksheet with a series of questions, including what their vision for a healthy Maasai community consisted of and what they perceived their role in achieving this to be. Prompts to guide the reflection included: access to clean water and toilets/latrines; food; access to a doctor/hospital/ healer; and physical, social, and spiritual health. The worksheet also included a section where they could use the arts and draw their vision. The sheets were completed by SHINE participants and, together with the sanitation mural and science fair posters, were put into a container that was buried under the ground at the school. After 5 years have passed, the intention is that the Project SHINE time capsule will be opened, and the students and community will be able to discuss and reflect on the changes that have taken place.

\subsubsection{Digital Stories}

Storytelling is deeply engrained in Maasai culture, and in order to tap into this mode of expression to capture and convey what it meant to participants to be involved in the project, digital stories were created in partnership between the research team and participants. Digital storytelling permits participants to build narratives about their experiences through different mediums such as video, audio, imagery, music, or text (Lambert and Hessler 2018). It was explained to participants that we wanted to use a technique that was culturally relevant to the Maasai to share the SHINE story to the wider world, and that using visual means and digital stories might be a useful tool for reflecting and sharing participant experiences.

The photos that were taken of the sanitation science fair by students with disposable cameras were printed and shared with students. In addition, photos taken by the research team were included among the options students could select from for the digital stories, so that there was a wide range of photographs to choose from to represent participant memories and experiences. Storyboarding sessions were facilitated by members of the University of Calgary team with guiding questions to help students organize the sequence of photos and develop captions for the digital stories.

When this activity was completed, those who wished their experiences and reflections to be captured on film were taken to an adjacent room for filming. Upon their return to Canada, the University of Calgary students, with the assistance of a filmmaker, finalized the digital stories and stored and shared them on USB devices 
with all participants and with the school as a token of appreciation for their participation in the study and to serve as a memory of the project. Although not all or even most students are likely to have a computer on which they can view the photos, they do have access to computers on the school premises, and the digital stories can be shared with parents at school meetings.

\subsection{Formative Research: Application of Photovoice in the Adaption of Project SHINE to an Indian Community}

Throughout India, poor sanitation and hygiene is a widespread public health challenge. Indians account for one-third of the 2.3 billion people worldwide who do not have access to improved sanitation in their home (World Health Organization and UNICEF 2017), and this is a leading contributor to the diarrheal disease burden of children under age five (Boschi-Pinto et al. 2008). Given India's vulnerability to diarrheal disease and other sanitation-related challenges, solutions that incorporate the country's social, cultural, and environmental context are in great demand. Project SHINE was a relevant health promotion intervention that contributed to the country's existing efforts to improve WASH-related health outcomes for communities. However, given India's rich cultural heritage and distinct historical legacy, a rigorous adaptation and translation process from the Tanzanian context was needed.

SHINE India evolved from mutual interests in water, sanitation, and hygiene conveyed by leadership from the locally based Sri Narayani Hospital and Research Centre in Sripuram, Thirumalaikodi, and Dr. Bastien facilitated the partnership. Once a partnership was formally established, an academic research team was formed including doctoral and master students from Norwegian academic institutions (Norwegian University of Life Sciences and University of Bergen), local schools, community stakeholders, and spiritual leadership. Implemented from June 2017-July 2018 in the rural community of Sripuram, Thirumalaikodi within the Vellore District of Tamil Nadu (see Fig. 9.1 for timeline), in SHINE India, artsbased research methods were incorporated into the study design as a critical component of the formative research phase and adaptation framework. The study protocol was approved by the Norwegian Centre for Data Research (reference number: 53162) in Norway and the Institutional Ethics Committee/Institutional Review Board at the Sri Narayani Hospital and Research Centre (reference number: 30/25/02/17) in India. As an initial step, a month-long photovoice sub-study was conducted at a local school, which aimed to engage adolescent students in a group process of critical reflection and dialogue to assist in the adaptation and translation of the intervention to the local cultural context. Rooted in the tradition of documentary photography, photovoice provided adolescent students with cameras so that they could accurately capture and share their community's assets, challenges, and needs in relation to issues concerning water, sanitation, and hygiene (Wang and Burris 1997). These photographs, accompanied by critical discussion and reflection, 
helped inform changes to the SHINE India adaptation that was subsequently implemented in their school.

Artistic expression when applied to CBPR approaches can trigger discussions that facilitate the co-creation of knowledge between researcher and participant. Although there are several approaches that can develop a researcher's understanding of the cultural context, we felt that using an arts-based method not only could help co-create knowledge, but could also encourage and promote genuine partnerships, participation, and engagement through discussion and intimate sharing. In order to embark on this process, we needed to understand students' everyday lives. Figure 9.4 demonstrates how photovoice was utilized in the formative research and adaptation processes to help increase the cultural relevance, appropriateness, and appeal to student participants (Viswanathan et al. 2004).

\subsubsection{Photovoice: An Effective and Engaging Tool for Adapting the SHINE Intervention}

In the photovoice sub-study, a purposive sampling strategy was used to recruit participants. Here, the school principal and teachers recruited ten students (three boys and seven girls) aged 13-15 through classroom announcements and the distribution of project brochures. The research team, school principal, and a local project coordinator worked closely with participants throughout the sub-study. The project coordinator was a local schoolteacher and attended school with the students daily. She was also available to discuss any issues about the project if students were uncomfortable or unsure how to communicate with the principal investigator. The project coordinator was not present in photo discussion sessions (PDS) so that

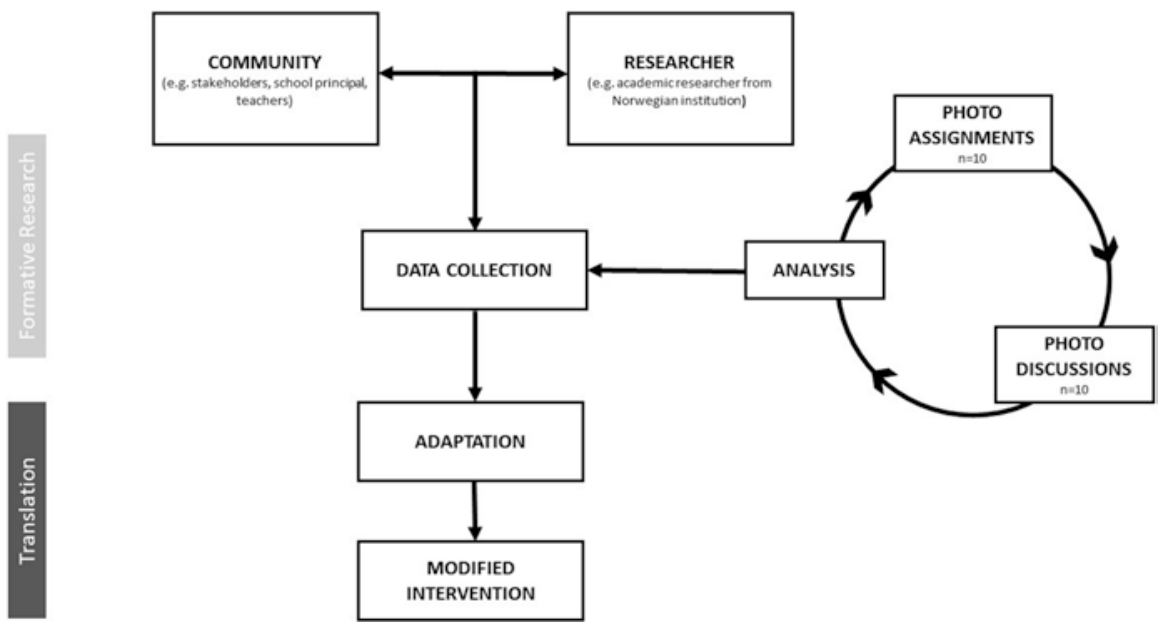

Fig. 9.4 Adaptation framework for Project SHINE India using arts-based method: Photovoice. (Adapted from McKleroy et al. 2006) 
students would not associate the photo discussions with a school assignment and would be able to communicate freely. Additionally, the project coordinator had regular meetings with the school principal to provide updates on the photovoice substudy progress and student feedback.

Participants attended one information meeting, seven PDS, and one wrap-up meeting over a three-week period. They were formally introduced to the photovoice sub-study in the initial information meeting, which the local project coordinator cofacilitated with the principal investigator. The main purposes of this information meeting were (1) for participants to become acquainted with the photovoice process and method, and (2) to build rapport between the participants and the principal investigator prior to in-depth PDS. Participants were also taught about photography ethics (see Box 9.1), how data and identities would be protected throughout research activities, and how to obtain informed written consent when taking photographs of people. They also had the opportunity to ask questions in English or in their native language, Tamil.

\section{Box 9.1 Photography Ethics: Implications and Procedures}

Susan Sontag discusses ethical implications embedded within the artistic medium of photography when she describes the camera as "a tool of power" (Sontag 2001); therefore, the first photovoice training included a discussion on photography and ethics. The technical processes involved when taking photos can offer a "permanent record" (Price 1994) or an "authentic" visual representation of the photographer's reality (Ball and Smith 1992; Collier and Collier 1986). However, when viewing a photograph, an individual may interpret it or react to it differently than the photographer intended (Killion 2001). Consequently, it is important that both researchers and participants discuss and reflect upon the power of taking pictures before they mutually decide to engage in a photovoice project. Since visual content can be easily objectified when revealed without context (Cooper and Yarbrough 2010; Frosh 2001), SHINE India participants were encouraged to take photos and narrate experiences from their own everyday lives (Cooper and Yarbrough 2010). Also, in this project, we discussed the importance of "storytelling" or providing context when sharing photographs. Guided by a handout and a short exercise in which students went through an example, the photovoice facilitator (PI) modeled how contextual descriptions can complement a photograph (such as descriptions of what the picture conveys and why the photographer chose to take this photo) to prevent misunderstandings and/or other misconceptions (Frosh 2001; Sontag 2001). Additionally, participants were carefully instructed that informed written consent must be obtained anytime they took a photograph of a person's face or any other identifiable feature because visible identifiers can easily connect individuals to an image, which has several other ethical implications in research. These consent processes were established to protect both the individuals in photographs and the photographers themselves (student participants) from harm, thus preventing issues such as unwanted attention, involuntary participation, marginalization, embarrassment, or shame. 
In this information meeting, participants were trained on how to use digital cameras. The group talked about their prior understanding of photography. Discussing the group's prior knowledge about the medium was an important step before distributing cameras, and it helped the facilitator structure the rest of the meeting based on the students' experience levels. Students expressed a major concern: is sanitation photogenic? The group had little to no experience using a camera, so we showed the group examples related to different research questions to help them conceptualize future assignments. During an explanation on the photovoice method, students browsed Visual Voices: 100 Photographs of Village China by the Women of Yunnan Province (Wu 1996) to see examples of photos from another photovoice project in China. After flipping through the book and seeing the different ways that these women photographed their community, students felt reassured and excited for the first photo assignment. It is important to consider that many students will reference advertisements or portraiture as the types of photographs that are customary in their environment. Here, it was crucial to bring examples of other ways to take photographs that illustrate reality or everyday life (e.g., newspaper clippings, postcards, books) so that students could see examples of different types of photography. We also discussed how to take photographs and photo aesthetics such as lighting, focus, and composition.

Once participants indicated that they understood the process, the first PDS were scheduled, and participants separated into single-sex groups to ensure that they were comfortable when talking about sensitive topics in front of their peers. After each photo assignment, each group would come together to share their photographs. All PDS followed the same procedure in both male and female groups; however, since the groups were conducted separately, the photo assignments generated differed. It is also important to note that participants were included and involved in the research question development processes, helping to ensure that the study genuinely addressed community-identified needs and interests.

Prior to each PDS, participants would select one or two photographs that they had taken for that day's photo assignment that they would like to share with the group. When the session started, each participant would share their selected photographs with the group. After all participants had shared their photographs, the entire group would vote on one photograph to discuss in depth.

To facilitate these in-depth discussions, we used an inductive mneumonic questioning technique known as the SHOWED method (questions include: what do you See; what is Happening; how does this relate to Our lives; Why does this situation/ strength exist; how can we become Empowered by our new understanding; what can we Do?) (Shaffer 1985). This method is based on Freirean processes of listening, dialogue, and action (Freire 1973, 2000) that were used to "trigger" critical dialogue and reflection (Wallerstein 1994). During discussions, the initial questions elicited critical dialogue first through description (See, Happening), then personalization (Our), while subsequent questions probed further critical analysis (Why), problem-posing (Empowered), and action (Do) (Wallerstein 1994).

It can be argued that the central focus of these discussions was not the photographs themselves, but the critical dialogue that ensued. For example, in one PDS a 


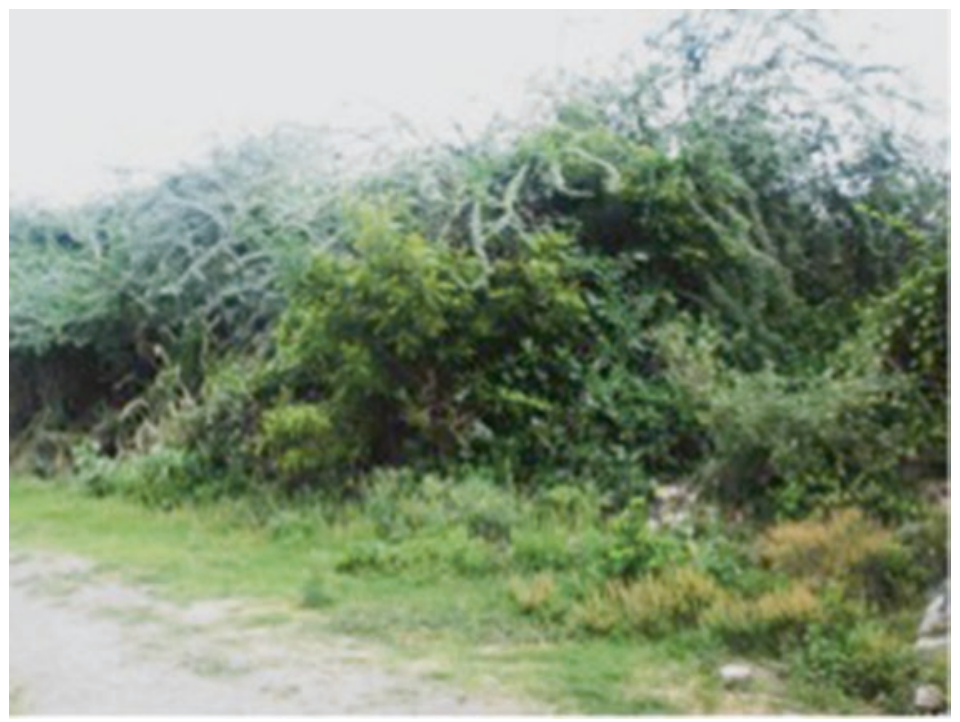

Fig. 9.5 Photovoice assignment 4: What habits do people have that are not hygienic?

participant shared a photograph (see Fig. 9.5) of a bush plant which generated an in-depth discussion about hygiene habits such as waste disposal and traditional health practices. Participants explained how this bush plant was not beneficial for their community because it grew thorns, produced no food, and did not have any medicinal value; therefore, people would dump waste on or around it. Then students began to discuss strategies for improving the community. For example, they spoke about how they could cut down the bush to prepare the land for planting medicinal herbs. This would perhaps sway people's attitudes about dumping their waste there.

\subsubsection{Shared Power: From Critical Dialogue to Action}

From the PDS, the academic research team identified key themes and applied them to a blend of adaptation frameworks traditionally used to translate evidence-based health promotion interventions such as Intervention Mapping (Bartholomew et al. 1998) and the Map of Adaptation Process (McKleroy et al. 2006). Using the photovoice data, these frameworks guided the intervention adaptation and knowledge translation of the SHINE school curriculum from Tanzania to the Indian cultural context. For example, students' photographs and discussions often revealed the cultural importance and sacredness of the natural/ecological environment, environmental sanitation, and waste management, which helped inform the translation of specific lessons in the SHINE Tanzania curriculum to have a more explicit focus on environmental sanitation and the natural environment for SHINE India. The adapted curriculum with the input from the photovoice sub-study was disseminated to over 
300 students in two schools in the SHINE India intervention in the local community of Sripuram, Thirumalaikodi.

\subsubsection{Project SHINE India Adaptation}

The main outcome of the photovoice project was to make changes in the SHINE India curriculum. Throughout India, many people do not use toilets and instead defecate out in the open because toilets are considered dirty and it is seen as defiling to clean an impure space (Lüthi 2010) such as a toilet. Students suggested in the PDS that they could help with cleaning and other household chores, therefore transforming social norms. According to social cognitive theory, social modeling is a powerful method of health behavior change (Bandura 1998; Glanz et al. 2008). Inspired by photovoice student participants, we decided to include a lesson in the intervention that included proper hygienic practices such as cleaning toilets and reusing waste.

\subsubsection{Photovoice Photograph Exhibition}

Similar to SHINE Tanzania, as part of the main intervention, students and teachers held a sanitation science fair where they came up with projects related to water, sanitation, and hygiene. Many students constructed models and performed demonstrations for their science fair projects. Parents, community members, and students from other classes were invited to tour the projects. In addition to the science fair, photovoice students decided that they would like to include their photographs in a display. This small photo exhibition was suggested by the local project coordinator and discussed during the final group meeting. Students saw an exhibition as an opportunity to display their work to their peers and community as well as promote awareness about key issues discussed during the project. The gallery of photographs and captions was displayed outside the sanitation science fair so that all attendees could view it before they entered the main exhibition.

\subsection{Reflections, Considerations, and Lessons Learned}

As arts-based research methods gain momentum, academic researchers and practitioners must also engage in continuous processes of self-reflection and selfevaluation (Minkler 2005; Minkler and Wallerstein 2003). Although these methods exhibit the potential to communicate and elicit discussion beyond traditional research praxis through various data collection and knowledge translation methods, we must also reflect on further methodological and epistemological limitations. Each SHINE intervention was implemented within distinct social, cultural, and environmental settings and populations. The following reflections illustrate the 
similarities and differences across these contexts while simultaneously unpacking the methodological dilemmas and experiences in practice concerning participant engagement, community partnerships, power, and empowerment.

\subsubsection{Meaningful Processes of Participant Engagement in Participatory Arts-Based Research}

Ensuring meaningful participant engagement was essential throughout both the formative research and implementation phases of both SHINE interventions. The inclusion of arts-based methods within SHINE contributed to broader and more authentic engagement of participants and community members because of the methods' capacity for equitable participation that encourages personal expression and sharing of one's lived experiences (Finley 2008). Often, this type of engagement contributed to critical reflection processes that addressed sensitive or stigmatized concerns (Switzer et al. 2015) and acted as an entry point for participants to engage in discussions concerning potentially sensitive health issues, such as open defecation and its effects on community health. Additionally, in the SHINE photovoice, students shared photographs of their everyday lives and spoke openly about their personal experiences with hygiene-related illnesses and disease in their community, thus contributing to the depth and richness of our understanding of the contextual setting. Moreover, in many instances, arts-based methods such as the sanitation mural in Tanzania facilitated an open dialogue and ensured that participant experiences and expertise remained at the center of the inquiry, while also helping balance power dynamics by providing opportunities for participants to share their perspectives in a way that resonates and is most relevant to their culture and context. However, engaging with arts-based methods is not without challenges, and upon reflection of our own positionalities and experiences, it is important to acknowledge and deconstruct the complexities of employing arts-based methods in health promotion research.

In both settings, a foundational component of the project was the collaborative efforts put forth by academic researcher-community partnerships; therefore, stakeholder inclusion in all phases of research was fundamental to the SHINE approach. While participatory arts-based methods were incorporated throughout the project to encourage authentic participation and equitable partnerships, we also collaborated with students, teachers, and the wider community beyond these methods in various forums such as think tanks, workshops, and focus groups in which participants, stakeholders, and researchers met and exchanged knowledge. In Tanzania, a think tank approach to engaging community stakeholders at the local hospital, schools, and wider community included traditional leaders, traditional birth attendants, outof-school youth, and parents in all phases of the piloting of SHINE, which supported equitable participation and community ownership of the intervention. While think tanks generated a space for community participants to articulate potential concerns about the intervention at each stage, the platform also encouraged the 
development of community-driven mitigation strategies, thus exemplifying colearning processes between academic researchers and community collaborators in which local knowledge and skill helped navigate challenges and structures that the research team might lack. Additionally, the evaluation teams for the sanitation science fairs, which consisted of a broad spectrum of community stakeholders, were highly engaged in the process of selecting culturally relevant strategies for improving WASH and subsequently served as an advisory board for the project. Their participation constitutes another means whereby the project incorporated a diverse cross-section of stakeholders with an aim to contribute to the overall sustainability of the project.

Although Project SHINE was deeply committed to developing equitable partnerships, it is also important to consider that systems of oppression, structural hierarchies, and power imbalances exist at the local community level, which may influence community-researcher partnerships. In India, although we held regular meetings with community stakeholders/gatekeepers who were involved with the local community-based organizations, hospitals, schools, and spiritual leadership, it is important to acknowledge that while diverse in their position and expertise, our collaborating partners do not represent all voices within the community. Although we may envision comprehensive community participation and representativeness, it may not be feasible within a complex community structure. Stakeholders can represent and communicate many concerns; however, we must not assume they are able to represent the entire community (Wallerstein and Duran 2006). The community is composed of a multitude of voices, and stakeholders/gatekeepers may not adequately represent certain segments of a community, such as the elderly and other hard-to-reach groups. Although we hoped to include the community in shaping the research project in order to ensure that the project addressed community-identified needs, it is important to recognize and reflect upon how each community has dynamic and complex historical, political, and cultural forces that shape knowledge and power. In SHINE India, community members such as parents and local elders were not directly involved in our formal stakeholder engagement process, though we did attempt to ameliorate this with informal interactions throughout our daily activities in the community. Sripuram, Thirumalaikodi is a community with a strong spiritual legacy, and engaging in this aspect of community life was central to our own investment in the partnership. This included daily participation in puja and regular participation in seva activities. We also chose to wear locally appropriate clothing and follow cultural norms and rituals regarding traditional activities. Participation in these activities was essential to showing our commitment and engagement in the community. However, formalized stakeholder meetings were especially important arenas of knowledge exchange, allowing us to report on current project activities and receive feedback on our progress. As these formal processes failed to reach certain segments of the community, such as the elderly or parents, we felt it was essential for the research team to build relationships outside of formalized space. To do so, we attended temple daily, attended school-based and religious or cultural celebrations, and greeted parents in the schoolyard to foster further engagement, trust, and mutual respect with community members. 


\subsubsection{The Potential for Arts-Based Methods as a Tool for Empowerment and Catalyst for Social Change}

One of the main aims of Project SHINE has been to encourage youth through the development of life and leadership skills. Powerlessness is a social determinant of health, and thus empowerment is essential to improving health outcomes. The World Health Organization (WHO) distinguishes between individual and community empowerment, defining individual empowerment in terms of an ability to make decisions and control one's personal life, while community empowerment is defined as collective action to "gain influence and control over the determinants of health and the quality of life in their community" (World Health Organization 1998). The goals of Project SHINE are both to aid in the participants developing life skills that lead to greater autonomy over their own health, and also to help them develop leadership skills that allow them to take part in leadership and civic engagement activities in the long term. With arts-based methods such as photovoice, time capsules, and murals, SHINE aimed to create a participatory platform that would foster engagement, ownership, and self-determination among the participants, which in turn would encourage the development of various life skills as well as self-confidence in their role as change makers in their communities (Wilson et al. 2007).

Project SHINE was developed as a CBPR project that creates a platform for data collection, art, and youth empowerment. Nevertheless, we should not assume that participation is inevitably empowering for the participants or co-collaborators, especially given the social structures surrounding youth participants. Action is a foundational component of CBPR, with participants engaging with stakeholders, gatekeepers, and policymakers to create social change. However, Strack et al. (2004) state that it is important to bear in mind that arts-based methods such as photovoice are a "process," and empowerment may not be completely realized by participants by the conclusion of a project or research study. Empowerment is a personal journey and likely a lifelong project for most people, and thus cannot be reduced to a simple measurable outcome of participation in a year-long study. Therefore, it is important to understand the value of processes of reflection and dialogue as crucial first steps in personal growth processes that lead to mobilization and empowerment, especially when working with youth who are undergoing intense experiences of personal growth and change (Strack et al. 2004).

While photovoice and other CBPR methods encourage participants to make contact with policymakers to create change in their communities, this may not always be a realistic goal, and a singular focus on this can be discouraging for the long-term success of a project (Johnston 2016). Both SHINE interventions were implemented in settings that had distinct social structures. In India, hierarchical social structures that value seniority shape systems and cultural norms that influence how youth are perceived, seen, and heard. In this context, social structures may also prevent students from having access to power within society. This leads to a question: to what extent can youth participants share their "voice"? In many countries around the world, youth are unable to vote or participate in civic organizations without consent 
from an adult. Even to participate in Project SHINE, all students needed to obtain parental consent. As we managed goals and expectations of the project, we also acknowledged that adolescents often must act through proxy and reflected upon local systems of power and processes of empowerment. However, this is not to suggest that empowerment cannot be achieved through skills development and feelings of self-confidence and self-worth; rather, it is to remind us that researchers must acknowledge potential benefits and limitations of methods in order to effectively facilitate and support project participants. Additionally, researchers engaged in artsbased CBPR research should be cautious when raising expectations of project participants. In the SHINE PDS, participants brainstormed solutions for identified challenges without limitations, such as a discussion on potential sanitation enforcement strategies that are punishable by fines, or imprisonment for individuals who are seen dumping waste in the community's water sources. However, society, power structures, corruption, and resources may limit participant control of everyday life and their abilities to implement such strategies.

\subsubsection{Tapping the Potential of Arts-Based Methods for Unlocking the Creativity and Curiosity of Youth in Health Promotion Interventions}

Arts-based methods were also integrated into SHINE to provide a platform for youth as social actors, future educators, scientists, and leaders and to enable them to express themselves in diverse ways that would resonate with their culture and context. The linguistic complexity of the setting was one factor in adopting a participatory arts-based method within the project. Although English is the language of instruction at secondary schools in Tanzania, as well as in our partner schools in India, it is not the participants' first language in either setting. Moreover, given the strong community focus within the project, the predominately oral culture among Maasai pastoralists, and potentially low literacy levels, including a more diverse spectrum of possibilities for expression within the project was important in terms of inclusion of diverse perspectives and modes of expression, as well as ensuring a broader reach and overall sustainability of the project.

We found students to be positively engaged in this component of the project, and it was frequently mentioned that they lacked a similar creative outlet within their school setting. Community members reflected that it was important that the knowledge created as part of the project be shared beyond the walls of the classroom and spread to the wider community, speaking to the potential of arts-based methods in health promotion to span the divide that often exists between schools and communities. Teachers similarly expressed appreciation for the inclusion of arts-based methods in the project, yet discussion indicated that within the national curriculum there is insufficient focus and space devoted to the arts. Similarly, the inclusion of arts such as dance and theater in SHINE India's knowledge translation and 
dissemination activities resonated with the local culture, where such performing arts are a common part of community life. After the photovoice sub-study, three students wrote and directed a short skit about the importance of water (a key takeaway from the photo discussion sessions and a community-identified health concern), while other students choreographed a dance about handwashing (see Fig. 9.6). Participants expressed that they felt happy sharing their knowledge and experiences with their peers through art.

\subsubsection{Can Arts-Based Methods Alleviate Power Imbalances?}

Power imbalances can exist within community-researcher, young-elder, and other superior-subordinate relationships that are replicated and reinforced by history, politics, culture, and tradition (Muhammad et al. 2015). In many ways, the inclusion of arts-based methods in Project SHINE was intended to mitigate power differentials in the research process. As mentioned above, the SHINE Tanzania intervention was implemented in a context of considerable linguistic complexity. The incorporation of arts-based methods was crucial to giving a broad and diverse platform for youth expression. Additionally, literacy levels among both students and community members could not be assured; therefore, providing a medium that nurtured personal expression and sharing was essential for equitable engagement.

However, despite efforts to minimize power differentials, we acknowledge several difficulties of establishing truly equitable partnerships. In the SHINE India project, the photovoice process aims to overcome imbalances in power in the relationship between participant and researcher; however, this is especially difficult when working with youth in a setting where distinct social structures are prominent. To overcome this, we tried to develop a supportive environment where we could learn together, with participants and their experiences as the focal point of all interactions. Although we had certain time constraints, we focused on building rapport and allowing space in all our conversations with participants for questions and debate. We discussed the participants' roles as co-researchers and the instrumentality of
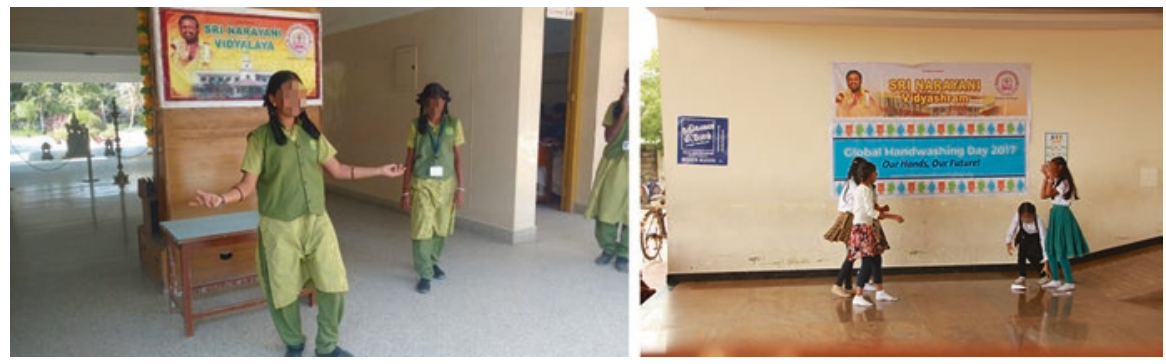

Fig. 9.6 Students in SHINE India engage in performing arts to teach peers about the importance of water (left) and handwashing (right) 
their experiences and concerns to the development of the research project. Through looking at the photographs and discussing the various issues they identified within their community, we started to develop new knowledge and understanding of the local context together. The cultural significance of the natural environment quickly emerged as important to the participants, alongside concerns about generational divides regarding the importance of ecological preservation. Exploring these ideas and concerns was essential to the adaptation of the SHINE curriculum to the Indian context. We continuously tried to communicate the implications and impact of the research and the importance of the participants' involvement. However, power imbalances between participants also posed a challenge to the equitability of the PDS experience. Janes (2016) discusses how in some ways CBPR can preserve power differentials in community-researcher partnerships despite the approach's claims to prevent and/or mitigate oppressive research practices. As only one photo is selected and discussed, dominant members of the group can end up controlling the direction of the discussion, and certain participants may not have their voices heard, potentially resulting in antagony (for background on this issue, see Chap. 21, this volume). To prevent this, we selected the photo for discussion through an anonymous vote and developed group norms aimed at creating an open, inclusive, and supportive environment in which everyone felt comfortable expressing their opinions during the initial photovoice meeting.

Through the application of a CBPR approach, the research project brought academic researchers, communities, and students together to exchange knowledge and disseminate findings. However, a research project developed with the intention to encourage equitable partnerships can also contribute to the very power dynamics it seeks to disrupt (Janes 2016). We hope to expose additional tensions related to power within this type of work to encourage a push toward more reflexive and transparent research praxis. As we build meaningful partnerships with community collaborators, it is crucial that we try to understand how research skills are applied in the local context, so these skills and capacity can be leveraged outside the research project to ensure that the project is mutually beneficial throughout the entirety of the research process. In Project SHINE, although participants were excited about the intervention itself, we experienced difficulties recruiting community collaborators to contribute to the academic writing processes (i.e., we have not been able to reach a teacher who was deeply involved in implementing SHINE Tanzania to co-author this chapter). Mindful of the potential threat of tokenism (Arnstein 1969)—which would consist of merely including names to give the appearance that we engaged the community-we used this opportunity to reflect and learn more about local ways of communicating and disseminating information, which often included visual and performing arts such as theater, song, and dance (described in previous sections). As collaborative partners, we should also explore alternative methods of dissemination, since academic publications may not represent "currency" to local counterparts or be accessible to collaborating communities. By limiting knowledge translation to the confines of academic tradition, we sustain the power and privilege of academics and diminish inclusive and equitable practices of the CBPR approach. Therefore, it is necessary to critically reflect on such challenges and limitations in 
an attempt to build the academic researcher's capacity to become more adept in understanding alternative communication methods and knowledge translation.

\subsection{Conclusion}

In Project SHINE, arts-based methods were used at various stages of the intervention to engage youth, teachers, and the wider community not only in the development of culturally relevant and sustainable strategies to improve water, sanitation, and hygiene, but also more broadly to promote youth leadership and to provide an additional avenue or platform for expression within the project. This demonstrates how arts-based methods can be integrated into health promotion interventions to increase authentic engagement, encourage empowerment processes, increase understanding, translate knowledge, and aid in knowledge translation to the wider community. However, from a methodological standpoint, systematic and thoughtful inclusion of arts-based methods at all phases of the research-alongside more traditional methods such as in-depth interviews, focus group discussions, and surveysis a promising strategy for meaningfully engaging youth and communities and enhancing triangulation within a study. The use of arts-based methods also allowed the researchers to gain a deeper understanding of the cultural contexts we were working in through engaging with personal and communal forms of artistic expression and encouraging active listening and continuing reflection throughout the process. In many ways, the incorporation of the arts was a continuous organic process which made it challenging to evaluate their effect and impact. Therefore, we recommend that similar interventions aiming to reach youth should systematically plan and sequence the incorporation of arts-based methods into an evaluation. This would help further strengthen legitimacy of methods and allow for effective and systematic evaluation of processes and outcomes.

Although each SHINE intervention incorporated different arts-based research methods during different phases of research to help achieve research project objectives under a CBPR frame, future iterations would need to develop meaningful indicators and apply systematic evaluation frameworks in order to realize the full potential of arts-based methods when applied to research. However, throughout these two interventions, we witnessed participants sharing their personal insights, reflections, and experiences through creative expression, which-despite criticisms of voice, power, and empowerment-did inspire a sense of responsibility to the community and improve our understanding of the health issues that were important to the community. Moreover, the inclusion of arts-based methods has fostered alternative methods of knowledge translation that markedly challenge us as academic researchers to disrupt and critique traditional academic praxis and work toward more equitable and creative approaches in future projects. Although the use of methods differed, their inclusion led to unique partnership engagement, knowledge sharing, and learning, demonstrating how these methods can be utilized in health interventions as a tool for education, knowledge translation, and action. 
Acknowledgements Grand Challenges Canada (Tanzania) and CINIM (India) funded this project.

We would like to thank all the schools, teachers, students, and community stakeholders in the Tanzanian and Indian communities who participated and contributed to this research project. In addition, we would like to acknowledge the contributions and support of Erika Laurel Friebe and "Godwin" Saningo Olemshumba (SHINE Tanzania) and Ramesh Shanmugasundaram, Nathalie Latham, Balaji Nandagopal, Sri Sakthi Amma, and photovoice participants (SHINE India).

\section{References}

Arnstein, S. R. (1969). A ladder of citizen participation. Journal of the American Institute of Planners, 35(4), 216-224.

Ball, M. S., \& Smith, G. W. (1992). Analyzing visual data. Thousand Oaks: Sage Publications.

Bandura, A. (1998). Health promotion from the perspective of social cognitive theory. Psychology and Health, 13(4), 623-649.

Barone, T., \& Eisner, E. W. (2012). Arts based research. Thousand Oaks: Sage Publications.

Bartholomew, L. K., Parcel, G. S., \& Kok, G. (1998). Intervention mapping: A process for developing theory and evidence-based health education programs. Health Education \& Behavior, 25(5), 545-563.

Bartram, J., Charles, K., Evans, B., O'hanlon, L., \& Pedley, S. (2012). Commentary on communityled total sanitation and human rights: Should the right to community-wide health be won at the cost of individual rights? Journal of Water and Health, 10(4), 499-503.

Bastien, S., Hetherington, E., Hatfield, J., Kutz, S., \& Manyama, M. (2016). Youth-driven innovation in sanitation solutions for Maasai pastoralists in Tanzania: Conceptual framework and study design. Global Journal of Health Education and Promotion, 17(1).

Bastien, S., Hetherington, E., Williams, K., Hatfield, J., \& Manyama, M. (2017). The development of an innovative one health sanitation science fair to cultivate change agent capacity among pastoralist youth in rural Tanzania. In S. Bastien \& H. Holmarsdottir (Eds.), Youth as architects of social change (pp. 77-95). Cham: Palgrave Macmillan.

Boschi-Pinto, C., Velebit, L., \& Shibuya, K. (2008). Estimating child mortality due to diarrhoea in developing countries. Bulletin of the World Health Organization, 86, 710-717.

Cahnmann-Taylor, M., \& Siegesmund, R. (Eds.). (2018). Arts-based research in education: Foundations for practice. New York: Routledge.

Campbell, S. (2012). Knowledge translation curriculum. Ottawa: Canadian Coalition for Global Health Research.

Coemans, S., \& Hannes, K. (2017). Researchers under the spell of the arts: Two decades of using arts-based methods in community-based inquiry with vulnerable populations. Educational Research Review, 22, 34-49. https://doi.org/10.1016/j.edurev.2017.08.003.

Collier, J., \& Collier, M. (1986). Visual anthropology: Photography as a research method. Albuquerque: University of New Mexico Press.

Cooper, C. M., \& Yarbrough, S. P. (2010). Tell me-Show me: Using combined focus group and photovoice methods to gain understanding of health issues in rural Guatemala. Qualitative Health Research, 20(5), 644-653.

Delgado, M. (2015). Urban youth and photovoice: Visual ethnography in action. New York: Oxford University Press.

Finley, S. (2008). Arts-based research. In Handbook of the arts in qualitative research: Perspectives, methodologies, examples, and issues (pp. 71-81). Thousand Oaks, CA: SAGE Publications.

Freire, P. (1973). Education for critical consciousness. London: Bloomsbury.

Freire, P. (2000). Pedagogy of the oppressed. New York: Continuum. 
Frosh, P. (2001). The public eye and the citizen-voyeur: Photography as a performance of power. Social Semiotics, 11(1), 43-59.

Glanz, K., Rimer, B. K., \& Viswanath, K. (2008). Health behavior and health education: Theory, research, and practice. San Francisco: Jossey-Bass.

Graham, I. D., Logan, J., Harrison, M. B., Straus, S. E., Tetroe, J., Caswell, W., et al. (2006). Lost in knowledge translation: Time for a map? Journal of Continuing Education in the Health Professions, 26(1), 13-24.

Henderson, R. I., Hatfield, J., Kutz, S., Olemshumba, S., Van Der Meer, F., Manyama, M., et al. (2016). 'We can't get worms from cow dung': Reported knowledge of parasitism among pastoralist youth attending secondary school in the Ngorongoro Conservation Area, Tanzania. Journal of Biosocial Science, 48(6), 746-766.

Hetherington, E., Eggers, M., Wamoyi, J., Hatfield, J., Manyama, M., Kutz, S., et al. (2017). Participatory science and innovation for improved sanitation and hygiene: Process and outcome evaluation of project SHINE, a school-based intervention in rural Tanzania. BMC Public Health, 17(1), 172.

Israel, B., Eng, E., Schultz, A., \& Parker, E. (2005). Methods in community-based participatory research for health. San Francisco: Jossey-Bass.

Janes, J. E. (2016). Democratic encounters? Epistemic privilege, power, and community-based participatory action research. Action Research, 14(1), 72-87.

Johnston, G. (2016). Champions for social change: Photovoice ethics in practice and 'false hopes' for policy and social change. Global Public Health, 11(5-6), 799-811.

Kar, K., \& Chambers, R. (2008). Handbook on community-led total sanitation. Brighton: Institute of Development Studies, University of Sussex; http://www.communityledtotalsanitation.org/ resource/handbook-community-led-total-sanitation. Accessed 10 June 2020.

Killion, C. M. (2001). Understanding cultural aspects of health through photography. Nursing Outlook, 49(1), 50-54.

Lambert, J., \& Hessler, H. B. (2018). Digital storytelling: Capturing lives, creating community. New York: Routledge.

Leavy, P. (2015). Method meets art: Arts-based research practice. New York: Guilford Publications.

Lohan, M., Aventin, Á., Oliffe, J. L., Han, C. S., \& Bottorff, J. L. (2015). Knowledge translation in men's health research: Development and delivery of content for use online. Journal of Medical Internet Research, 17(1).

Lüthi, D. (2010). Private cleanliness, public mess: Purity, pollution and space in Kottar, South India. In E. Dürr \& R. Jaffe (Eds.), Urban pollution: Cultural meanings, social practices (pp. 57-85). New York: Berghahn Books.

McEwan, A., Crouch, A., Robertson, H., \& Fagan, P. (2013). The Torres indigenous hip hop project: Evaluating the use of performing arts as a medium for sexual health promotion. Health Promotion Journal of Australia, 24(2), 132-136.

McKleroy, V. S., Galbraith, J. S., Cummings, B., Jones, P., Harshbarger, C., Collins, C., et al. (2006). Adapting evidence-based behavioral interventions for new settings and target populations. AIDS Education and Prevention, 18(4), 59-73. https://doi.org/10.1521/aeap.2006.18. supp.59.

Minkler, M. (2005). Community-based research partnerships: Challenges and opportunities. Journal of Urban Health, 82(2), ii3-i12.

Minkler, M., \& Wallerstein, N. (Eds.). (2003). Community based participatory research for health. San Francisco: Jossey-Bass.

Muhammad, M., Wallerstein, N., Sussman, A. L., Avila, M., Belone, L., \& Duran, B. J. C. S. (2015). Reflections on researcher identity and power: The impact of positionality on community based participatory research (CBPR) processes and outcomes. Critical Sociology, 41(7-8), 1045-1063. https://doi.org/10.1177/0896920513516025.

Pattanayak, S. K., Yang, J.-C., Dickinson, K. L., Poulos, C., Patil, S. R., Mallick, R. K., et al. (2009). Shame or subsidy revisited: Social mobilization for sanitation in Orissa, India. Bulletin of the World Health Organization, 87(8), 580-587. 
Price, M. (1994). The photograph: A strange confined space. Palo Alto: Stanford University Press. Shaffer, R. (1985). Beyond the dispensary. Nairobi: African Medical and Research Foundation.

Sontag, S. (2001). On photography. New York: Macmillan.

Strack, R. W., Magill, C., \& McDonagh, K. (2004). Engaging youth through photovoice. Health Promotion Practice, 5(1), 49-58.

Straus, S. E., Tetroe, J., \& Graham, I. (2009). Defining knowledge translation. CMAJ, 181(3-4), $165-168$.

Switzer, S., Guta, A., de Prinse, K., Carusone, S. C., \& Strike, C. (2015). Visualizing harm reduction: Methodological and ethical considerations. Social Science \& Medicine, 133(SI), 77-84.

Vindrola-Padros, C., Martins, A., Coyne, I., Bryan, G., \& Gibson, F. (2016). From informed consent to dissemination: Using participatory visual methods with young people with long-term conditions at different stages of research. Global Public Health, 11(5-6), 636-650.

Viswanathan, M., Ammerman, A., Eng, E., Gartlehner, G., Lohr, K. N., Griffith, D., et al. (2004). Community-based participatory research: Assessing the evidence: Summary. AHRQ evidence report summaries (pp. 1-8). Rockville: Agency for Healthcare Research and Quality.

Wallerstein, N. (1994). Empowerment education applied to youth. Multicultural Challenge in Health Education, 161-162.

Wallerstein, N., \& Duran, B. (2006). Using community-based participatory research to address health disparities. Health Promotion Practice, 7(3), 312-323.

Wang, C., \& Burris, M. A. (1997). Photovoice: Concept, methodology, and use for participatory needs assessment. Health Education \& Behavior, 24(3), 369-387.

Wang, C., Yi, W. K., Tao, Z. W., \& Carovano, K. (1998). Photovoice as a participatory health promotion strategy. Health Promotion International, 13(1), 75-86.

Wang, Q., Coemans, S., Siegesmund, R., \& Hannes, K. (2017). Arts-based methods in socially engaged research practice: A classification framework. Art/Research International: A Transdisciplinary Journal, 2(2), 5-39.

Wilson, N., Dasho, S., Martin, A. C., Wallerstein, N., Wang, C. C., \& Minkler, M. (2007). Engaging young adolescents in social action through Photovoice-The youth empowerment strategies (YES!) project. Journal of Early Adolescence, 27(2), 241-261. https://doi. org/10.1177/0272431606294834.

World Health Organization. (1998). Health promotion glossary. https://www.who.int/healthpromotion/about/HPR\%20Glossary\%201998.pdf?ua=1. Accessed 10 June 2020.

World Health Organization, \& UNICEF. (2017). Progress on sanitation and drinking water: 2017 update and SDG baselines.

Wu, K. (1996). Visual voices: 100 photographs of village China by the women of Yunnan Province. Yunnan: Yunnan People's Publishing House.

Zinsstag, J., Schelling, E., Waltner-Toews, D., \& Tanner, M. (2011). From "one medicine" to "one health" and systemic approaches to health and well-being. Preventive Veterinary Medicine, 101(3-4), 148-156.

Open Access This chapter is licensed under the terms of the Creative Commons AttributionNonCommercial-NoDerivatives 4.0 International License (http://creativecommons.org/licenses/ by-nc-nd/4.0/), which permits any noncommercial use, sharing, distribution and reproduction in any medium or format, as long as you give appropriate credit to the original author(s) and the source, provide a link to the Creative Commons license and indicate if you modified the licensed material. You do not have permission under this license to share adapted material derived from this chapter or parts of it.

The images or other third party material in this chapter are included in the chapter's Creative Commons license, unless indicated otherwise in a credit line to the material. If material is not included in the chapter's Creative Commons license and your intended use is not permitted by statutory regulation or exceeds the permitted use, you will need to obtain permission directly from the copyright holder. 